\title{
Body Mass Index Finding
}

National Cancer Institute

\section{Source}

National Cancer Institute. Body Mass Index Finding. NCI Thesaurus. Code C138901.

The result of a body mass index measurement. 